\title{
Nano Co-crystal Engineering Technique to Enhance the Solubility of Ezetimibe
}

\author{
Jyothi Bhandari', Neha Kanswami², Lakshmi P.K* \\ 'Department of Pharmaceutics, St. Paul College of Pharmacy, Hyderabad, Telangana, INDIA. \\ 2Department of Pharmaceutics, G. Pulla Reddy College of Pharmacy, Mehdipatnam, Hyderabad, Telangana, INDIA.
}

\begin{abstract}
Background: Co-crystals have been highly promising for tailoring physicochemical properties of Active pharmaceutical ingredient (API) by coupling with co-former. Objectives: The objective of the present work was to prepare and characterize novel nano co-crystals of Ezetimibe by different methods in various ratios of co-formers and to optimize the formulation based on the enhancement in solubility and dissolution rate. Methods: Ezetimibe nano co-crystals were prepared employing oxalic acid, succinic acid and maleic acid as co-formers by solvent evaporation method and anti-solvent method. Results: Instrumental analysis of co-crystals (DSC, $I R, S E M$ and $X R D$ ) was performed to characterize the novel nano co-crystals. Dissolution studies and chemical stability were assessed and compared with pure Ezetimibe. The formulation with maleic acid as a co-former in the molar ratio of Ezetimibe and maleic acid $(0.4: 0.4)$ was found to be efficient than oxalic acid and succinic acid. The co-crystal dissolution profile in distilled water containing $0.5 \%$ SLS showed 18.8 folds increase in the
\end{abstract}

dissolution efficiency and was found to be $95.2 \%$ within 45 min. Conclusion: The results demonstrate feasibility of co-crystallization method using maleic acid as co-former to enhance the solubility of poorly soluble drug Ezetimibe.

Key words: Anti-solvent, Co-Formers, Maleic Acid, Oxalic acid, Solubility, Solvent evaporation, Succinic acid

\section{Correspondence}

Dr. P. K. Lakshmi,

Professor and Head, Department of Pharmaceutics, G. Pulla Reddy College of Pharmacy, Affiliated to Osmania University, Hyderabad-500028, Telangana, INDIA.

Phone: +9000044452

Email: drlakshmisuresh@gmail.com

DOI: 10.5530/jyp.2020.12s.40

\section{INTRODUCTION}

The oral route is the most preferred route of drug administration due to its convenience, good patient compliance and low medicine production costs. In order to a drug to be absorbed into the systemic circulation following oral administration, the drug must be dissolved in gastric fluids. For hydrophobic drugs, the dissolution process acts as the rate controlling step, which determines the rate and degree of absorption. Thus, one of the major challenges to development today is poor solubility, as an estimated $40 \%$ of all newly developed drugs are poorly soluble or in soluble in water. ${ }^{1}$

Bioavailability of poorly water soluble hydrophobic drugs (class II and class IV in biopharmaceutical classification system) is limited by solubility and dissolution rate. The dissolution rate of these drugs can be improved by decreasing particle size, decreasing crystallinity and/or increasing surface area. Several studies have been carried out to increase the rate of drugs dissolution by increasing the particle size. However, the fine drug particles have high tendency to agglomerate due to Vander Waals attraction or hydrophobicity, which both result in decrease in surface area over time., ${ }^{2,3}$

The enhancement of oral bioavailability of such poorly water soluble drugs remains one of the most challenging aspects of drug development. The development of nano co-crystals as a practically viable method to enhance bioavailability of poorly water soluble is to overcome the limitations of previous approaches such as salt formation, solubilization by co-solvents, particle size reduction and solid dispersion. ${ }^{4}$

One of the challenging tasks in the pharmaceutical industry is to discover ways of improving the physicochemical properties of active pharmaceutical ingredients (APIs). The solubility, dissolution rate, melting point, moisture sorption tendency and compressibility of APIs and/or recipients affect the bioavailability, design, processing, manufacturing and stability of the resultant dosage form. ${ }^{5}$ Pharmaceutical co-crystals are attractive to the pharmaceutical industry because they offer multiple opportunities to modify the chemical and/or physical properties of an API without making or breaking covalent bonds.

Co-crystals may be defined as crystalline materials that consist of two or more molecular species held together by non covalent forces. In the recent years Pharmaceutical nano co crystals are highly promising in enhancing the dissolution rates and thus, improved bioavailability and efficacy of medication. ${ }^{6}$ In pharmaceutical industry, it has been a major lucrative wherein the solid properties of pharmaceutical active agents have been modulated using complementary molecules in the form of cocrystalformers (CCFs). Co-crystalscontaininganactivepharmaceutical ingredient (API) can improve the physiochemical properties such as solubility/dissolution rate, stability and mechanical properties of an API. Nano-scaling will further advance these characteristics compared to their conventional forms because of a larger surface to volume ratio of nano sized particles, one can further improve properties of an API (e.g. dissolution rate). The enhanced dissolution rate of a nanocrystal is mainly due to the increased surface area. A slight increase in solubility owing to the curvature and the high-energy surfaces of nanosized particles will also contribute to faster dissolution. The components in a co-crystal exist in a definite stiochiometric ratio and assemble via noncovalent interactions such as hydrogen bonds, ionic bonds, - or Vander Waals interactions rather than by ion pairing. Further, co-crystals have

This is an open access article distributed under the terms of the Creative Commons Attribution-NonCommercial-ShareAlike 4.0 License, which allows others to remix, tweak, and build upon the work non-commercially, as long as the author is credited and the new creations are licensed under the identical terms. 
different crystal structures than the pure components, contain different intermolecular packing patterns and as such they often exhibit widely different physical properties than the pure components.?

\section{MATERIALS AND METHODS}

\section{Materials}

Ezetimibe was gifted by Lupin Pharmaceuticals Ltd., whereas Oxalic acid, Maleic acid, Succinic acid, Methanol and Ethyl acetate was gifted by SD-Fine chemicals.

\section{METHODOLOGY}

\section{Preparation of Nano Co-crystals of Ezetimibe by Solvent evaporation method}

Drug and the co-former (oxalic acid, succinic acid, maleic acid) in both $\mathrm{w} / \mathrm{w}(1: 0.25,1: 0.50,1: 0.75,1: 1)$ and $\mathrm{mmol}$ in a ratio of $(0.1: 0.1,0.2: 0.2$, 0.3:0.3 and 0.4:0.4) were dissolved in desired amount of ethyl acetate. This mixture was kept on hot plate at $75^{\circ} \mathrm{C}$ with continuous stirring. After getting clear solution the mixture was kept aside for two days. Nano co-crystals thus formed were collected and crystals were stored away from light and moisture.

\section{Preparation of nano cocrystals of ezetimibe using antisolvent method ${ }^{7}$}

Drug and the co-former (oxalic acid, succinic acid, maleic acid) in both $\mathrm{w} / \mathrm{w}(1: 0.25,1: 0.50,1: 0.75,1: 1)$ and $\mathrm{mmol}$ in a ratio of $(0.1: 0.1,0.2: 0.2$, 0.3:0.3 and 0.4:0.4) were separately dissolved in methanol. This solution was injected slowly into anti-solvent (water) at room temperature with moderate stirring. The formed product was dried at room temperature for 2 days. The formed Nano co-crystals was collected and stored away from light and moisture.

\section{Evaluation of Nano Co-Crystals Solubility studies}

Saturated solutions of samples were prepared and these samples were kept in orbital shaker for $24 \mathrm{hrs}$. After $24 \mathrm{hr}$ the samples were centrifuged for 15-20 min and then they were analyzed at $233 \mathrm{~nm}$ (UV).

\section{Solubility studies of pure drug in purified water \\ Solubility of ezetimibe in purified water was determined.}

\section{Dissolution}

Dissolution studies were performed with prepared nano co-crystals using USP II (paddle) apparatus in distilled water containing 0.5\% SLS, at a temperature of $37.5^{\circ} \mathrm{C} ; 75 \mathrm{RPM}$. Samples were withdrawn at regular intervals and were analyzed at $233 \mathrm{~nm}$ (UV).

\section{Assay}

Assay of prepared nano co-crystals were determined in distilled water. Accurately weighed amount of nano co-crystals equivalent to $10 \mathrm{mg}$ of drug was taken in $100 \mathrm{ml}$ volumetric flask, $20 \mathrm{ml}$ of methanol was added and shaken to dissolve the drug. The volume was made up to $100 \mathrm{ml}$ with distilled water. These were filtered and $1 \mathrm{ml}$ of aliquot of the above solutions were taken and diluted to $10 \mathrm{ml}$ with distilled water. The absorbance of these solutions was determined at $233 \mathrm{~nm}$ against blank. The percentage assay was calculated from the standard curve.

\section{Drug- Excipient Compatibility Studies}

The optimized formulations were evaluated for drug Excipient interaction studies via differential scanning calorimetry (DSC), X-ray diffractometry (XRD) and Fourier transformer Infrared spectroscopy (FTIR).

\section{Particle size}

The particle size of optimized formulation SE8 was determined by diluting or redispersing the samples with deionized water to ezetimibe concentration of $0.2 \mathrm{mg} / \mathrm{ml}$ prior to determination. The particle size was measured at $25^{\circ} \mathrm{C}$ using light scattering by Malvern Zetasizer. The polydispersity index was used to measure the size distribution. Measurement was performed in triplicates and the mean values were recorded.

\section{Differential Scanning Calorimetric}

The thermal properties of samples were characterized by DSC on SHIMADZU DSC-60 differential scanning calorimeter (Japan). Samples were placed on the non-hermetic aluminum pans. The sample cell was equilibrated at $25^{\circ} \mathrm{C}$ and heated at a rate of $10^{\circ} \mathrm{C} / \mathrm{min}$ over the range of $25-250^{\circ} \mathrm{C}$.

\section{Fourier Transform Infrared Spectroscopy}

SHIMADZU 8400s FTIR Spectrophotometer (Japan) was used for the characterization of samples and KBR pellet method was used for the characterization

\section{X-ray diffraction (XRD)}

XRD analysis was performed using SHIMADZU XRD 7000 (Japan). Shimadzu X-ray diffract meter XRD-7000 is a compact and general purposed X-ray. Diffract meter equipped with a - gone meter as standard. The goniometry is scanned in vertical direction with high precision in angle, so it makes us capable to measure various types of samples, the powder samples, thin film samples are hard to set and the samples are easy to dissolve by heat. The data processing software work under the latest OS "Windows.NT/2000".

\section{Analytical methodology for estimation of ezetimibe}

The analytical method for estimation of ezetimibe for the determination of its max and quantification of nano co-crystals before proceeding the experiment. Drug concentration of $30 \mu \mathrm{g} / \mathrm{ml}$ was scanned in distilled water with $0.5 \%$ SLS. An absorption maximum of $233 \mathrm{~nm}$ is obtained. The standard graph of ezetimibe in distilled water with $0.5 \%$ SLS was prepared at this max.

\section{RESULTS}

\section{Evaluation of Nano Co-Crystals}

Nano co-crystals were prepared by two methods. They are solvent evaporation method, anti-solvent method according to their formulation codes. These are evaluated for solubility, assay and dissolution studies.

\section{Assay}

The assay of optimized ezetimibe SE8 ezetimibe, maleic acid co-crystals (mmol) (0.4:0.4) was performed and was found to be $99.4 \%$ pure.

\section{Solubility studies}

Solubility of ezetimibe in purified water was found to be $0.072 \mathrm{mg} / \mathrm{ml}$. With the solvent evaporation method maximum solubility of $1.36 \mathrm{mg} /$ $\mathrm{ml}$ was observed with the drug. It has been observed that as the particle size decreases the solubility of ezetimibe ( $\mathrm{mmol}$ ) increases in solvent evaporation method. This may be due to the formation of hydrogen bonds between the drug and maleic acid. ${ }^{8}$ hence solvent evaporation method was optimized.

Solubility studies of ezetimibe was performed by increasing the amount of maleic acid and it was observed that even after increasing the amount of maleic acid, solubility of ezetimibe was not increased. Hence SE8 formulation was optimized. 


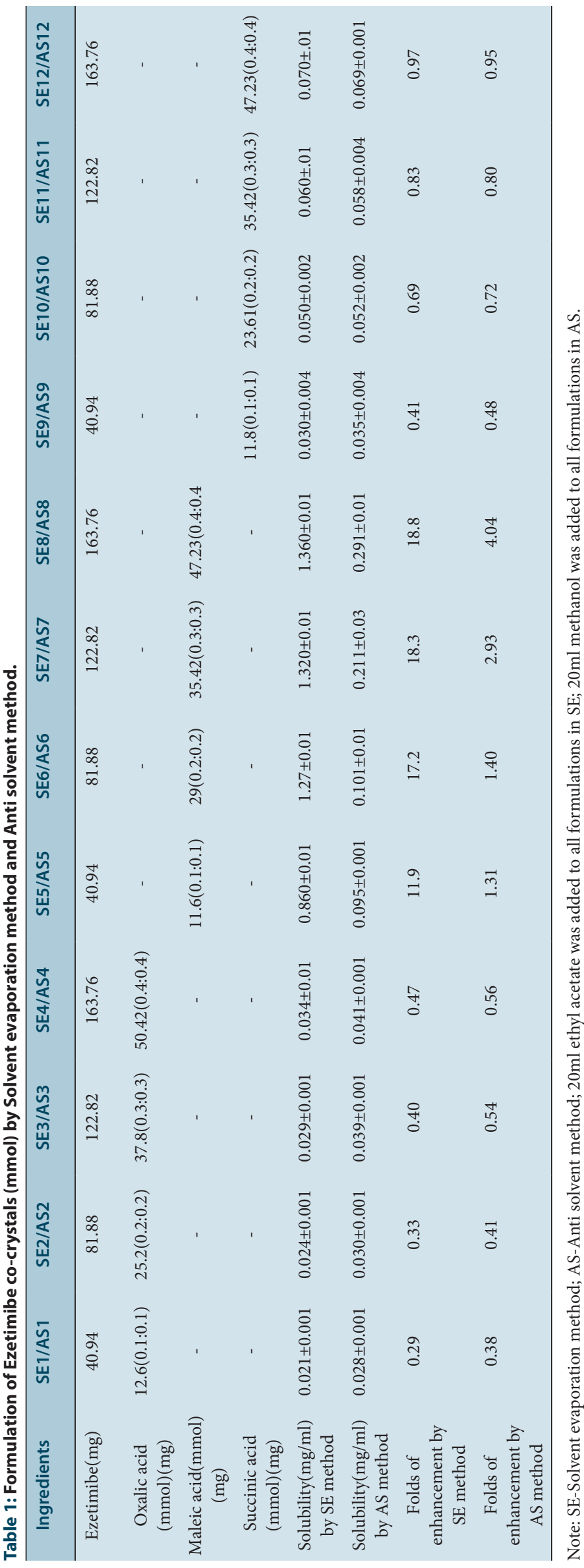

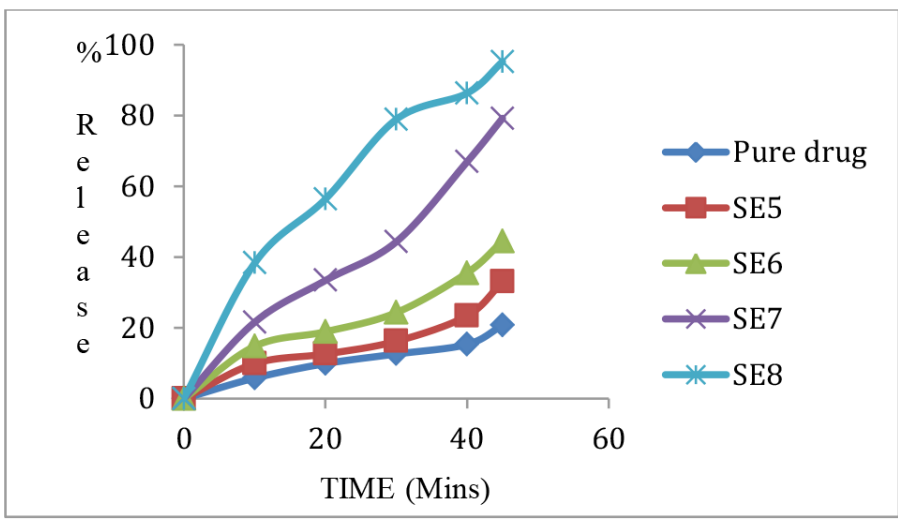

Figure 1: Percentage release profile of Ezetimibe nano co-crystals containing maleic acid in $0.5 \%$ SLS.

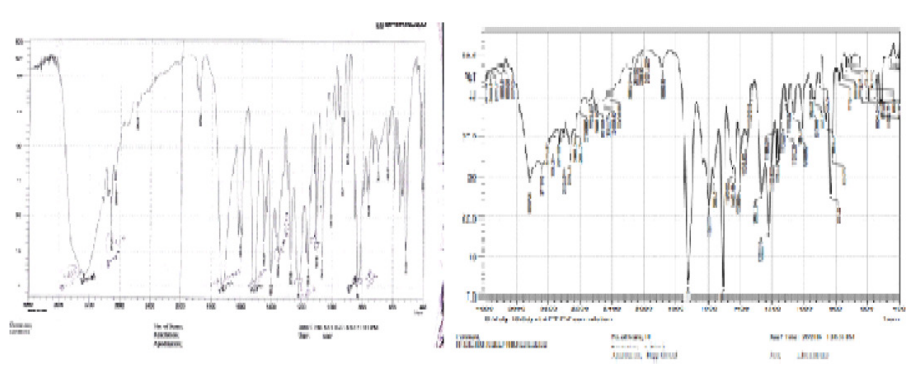

Figure 2: FTIR of Ezetimibe; FTIR of formulation SE8.

Note: SE8= Ezetimibe-Maleic acid co-crystals

\section{Dissolution studies}

Dissolution studies of all the formulations were performed according to the parameters the given Ezetimibe has shown only $20 \%$ release in $45 \mathrm{~min}$. this was observed due to the poor solubility of the drug which belongs to BCS class II. ${ }^{9}$

\section{In-vitro dissolution profile of nano co-crystals by Solvent evaporation method}

Dissolution studies of all the formulations were performed according to the parameters. Formulation containing $0.4 \mathrm{Mmol}$ (drug and co-former ratio) showed maximum release of $95.2 \%$ in $45 \mathrm{~min}$.

\section{Interaction studies of Ezetimibe by FTIR}

FTIR studies were done to verify if there was any interaction between the pure drug and various co-formers employed. The FTIR graphs, with drug and various co-formers were mixed and the blend was formulated into IR pellet and scanned. The different plots are given below.

- $\quad$ FTIR spectra of Ezetimibe and maleic acid have bands at $3266.29 \mathrm{~cm}^{-1}$ and $3058.89 \mathrm{~cm}^{-1}$ which are corresponding to $(\mathrm{O}-\mathrm{H})$ respectively which have been shifted to 3279.10 in the co-crystals formed .

- The bands at $1721.32 \mathrm{~cm}^{-1}$ of Ezetimibe and $1706.86 \mathrm{~cm}^{-1}$ of maleic acid which are corresponding to $(\mathrm{C}=\mathrm{O})$ respectively have been shifted to $1730.21 \mathrm{~cm}^{-1}$ in the co-crystals formed.

- The bands at $1446.51 \mathrm{~cm}^{-1}$ of Ezetimibe which is corresponding to (C-N) has been shifted to $1450.52 \mathrm{~cm}^{-1}$ which is likely due to the charge transfer of interaction between tertiary nitrogen of Ezetimibe with maleic acid. 
- These peak shifts strongly indicates the formation of hydrogen bond and other weak interactions between the Ezetimibe and maleic acid.

\section{Particle size}

The particle size of prepared nano cocrystals was found to be $226.4 \pm 53 \mathrm{~nm}$ and had a polydispersity index value of 0.540 .

\section{Thermal Analysis By Dsc}

Pure drug and formulation were subjected for DSC analysis to evaluate the change in crystalline of formulations. DSC thermo grams are illustrated through following figures

From the DSC analysis, it was observed that the thermo grams of the co-crystals Figure 3 . are showing melting point $\left(130^{\circ} \mathrm{C}\right)$ that is less than the API. This difference in pattern can be attributed to formation of eutectic, melting at a lower temperature than either of the two pure components drug $(163.5 \mathrm{C})$ and maleic acid $\left(135^{\circ} \mathrm{C}\right)^{10}$ From the results it appears that co-crystals obtained from API may be promoting additional co-crystallization and therefore maximizing the yield of co-crystals.

\section{Crystallinity by XRD}

Pure API and co-crystal formulation subjected for XRD analysis and following figures are their respective XRD graphs.

Co-crystal formation was confirmed by the presence of characteristic ezetimibe peaks at $15.6^{\circ}, 17.1^{\circ}, 18.6^{\circ}, 19.2^{\circ}, 20.8,21.6^{\circ}, 22.6^{\circ}, 23.3^{\circ}, 25.1^{\circ}$, $28.0^{\circ}$ and $29.9^{\circ}$ which are according to the previous literatures and were observed with intense peak at 19.24 .

Ezetimibe - maleic acid co-crystals in Figure 4 exhibited a unique XRD pattern that allowed them to be distinguished from Ezetimibe and maleic acid individually. It had peaks at the following 2 angles $18.5^{\circ}, 18.8,19.4^{\circ}, 20.7^{\circ}, 21.1^{\circ}, 21.8^{\circ}, 22.6^{\circ}, 23.2^{\circ}, 23.5^{\circ}, 24.3^{\circ}, 25.2^{\circ}, 26.2^{\circ}$, $27^{\circ}, 27.7^{\circ}, 28.1^{\circ}$ and $30^{\circ}$ and were observed with intense peak 27.7 .

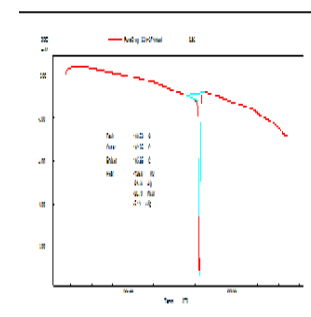

DSC of Pure drug

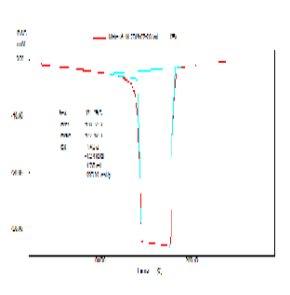

DSC of Vlaleic acid

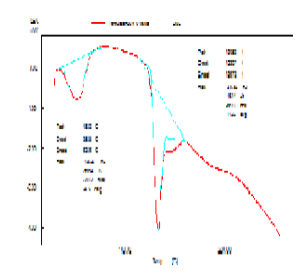

DSC of formulation SE8
Figure 3: DSC curve of pure drug; Maleic acid; formulation SE8.

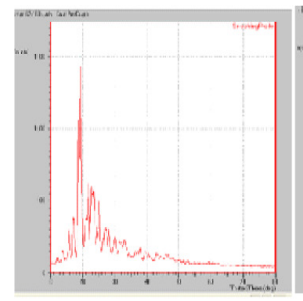

XRD pattern of pure drug

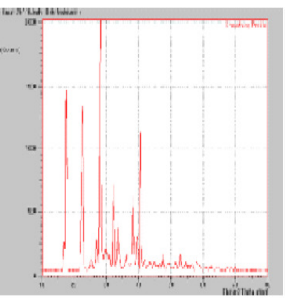

$\mathrm{XRD}$ pattern of maleic acid

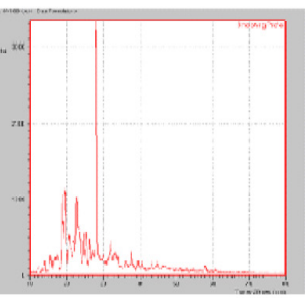

XRD pattern of formulation SE8

Figure 4: XRD patters of pure drug; Maleic acid; formulation SE8.

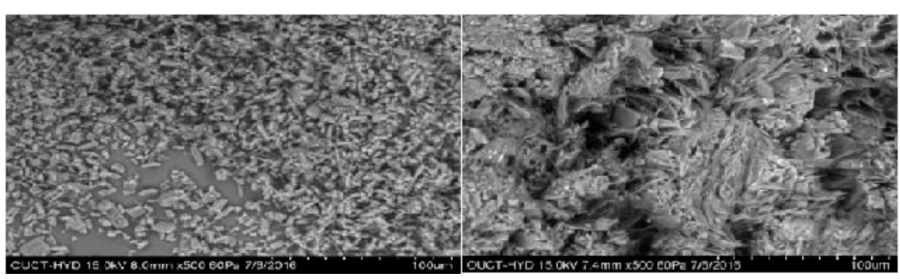

Figure 5: SEM Image of Ezetimibe; SEM Image of Formulation SE8.

Major peak at $19.2^{\circ}$ of pure Ezetimibe Figure 4 was missing in the co-crystal phase, whereas, peak at $27.7^{\circ}$ was observed. This confirms the formation of a new co-crystal phase. The probable conclusion which can be drawn from the XRD results is that the process facilitates co-crystallization. ${ }^{11}$

\section{SEM Results}

Surface morphology was studied using scanning electron microscope. Significant changes in the surface morphology were observed due to formulation process. Below mentioned figures represents the SEM results. Ezetimibe, as obtained from the supplier featured smaller particles as shown in Figure 5. The drug- maleic acid co-crystals formed from conventional sized ezetimibe were irregular shaped flake structures, as shown in Figure 5. Some of the crystals featured sharp edges while others had less defined edges and more irregular shape. Different surface morphology for co-crystals can be attributed to the particle size of API chosen for the formulations which in turn have influence on the crystal lattice during crystallization.

\section{DISCUSSION}

Solubility enhancement of Ezetimibe was performed by using nanococrystal engineering technique by using two different methods i.e, by using solvent evaporation method and anti-solvent method.

Ezetimibe - oxalic acid, maleic acid and succinic acid are connected by $\mathrm{N}$... H hydrogen bonds between the basic $\mathrm{N}$ atom of Ezetimibe and $\mathrm{H}$ atom of oxalic acid, maleic acid and succinic acid. Supra molecular synthons that can occur in common functional group such as carboxylic acids, amidesandalcoholsareparticularlyamenabletoformsupramolecular hetero synthons. The strong hydrogen bond includes (N-H...O), $(\mathrm{O}-\mathrm{H} \ldots \mathrm{O}),(\mathrm{N}-\mathrm{H} \ldots \mathrm{N})$ and $(\mathrm{O}-\mathrm{H} \ldots \mathrm{N})$. The weak hydrogen bond includes $-\mathrm{C}-\mathrm{H}$...O and $\mathrm{C}-\mathrm{H} . . . \mathrm{O}=\mathrm{C}$.

Ezetimibe is containing two hydrogen bond donors and three hydrogen bond acceptors. ${ }^{12}$ Two molecules of ezetimibe - maleic acid are connected by strong $\mathrm{N}-\mathrm{H}$... O hydrogen bonds forming a hydrogen bond dimer in a cyclic motif, ${ }^{13}$ which indicates potential for co-crystal formation based on molecular hydrogen bonding. Maleic acid being strong hydrogen bond acceptor and receptor was selected as the co-former to prepare co-crystals. Co-crystals of nevirapine prepared by using maleic acid as a co-former the aqueous solubility was enhanced ${ }^{14,15}$ and Co-crystals of exemestane and maleic acid showed high dissolution rate. ${ }^{16}$

Solvent evaporation method in mmol (0.4:0.4) using maleic acid as a co-former was observed to enhance the solubility of ezetimibe upto 18.8 folds compared to anti-solvent method in mmol (0.4:0.4) basis and this is because, for co-crystals solubility increases with the solubility of co-crystal conformers, co-former transition concentration increases with co-crystal and co-former solubilities, ${ }^{17}$ maleic acid being highly 
water soluble enhances the solubility of the co-crystals compared to Ezetimibe pure drug.

A check was done by increasing the amount of maleic acid in mmol, but it was observed that though the amount of maleic acid there was no further increase in the solubility of ezetimibe.

In anti-solvent method, both methanol and water are polar, both molecules exhibit hydrogen bonding where the hydrogen in one molecule is weakly covalently bonded to the oxygen in an adjacent molecule. ${ }^{17}$ The co-crystals prepared by this anti-solvent method demonstrated high solubility than that of pure drug because of the addition of anti solvent reduces the solute solubility in the resultant system. ${ }^{11}$ Out of the three co-formers used in this process, co-crystal prepared using maleic acid as a co-former showed better solubility than that of oxalic and succinic acid. Maleic acid has a higher dipole moment which enables intermolecular bonding with water molecules. ${ }^{14}$ But the dissolution profile of maleic acid was decreased in this method.

The dissolution data was evident that the pure drug was showing slow release than the formulation containing a co-former. The formulation prepared by solvent evaporation method showed a good release in 45 min compared to the pure drug and anti-solvent method, suggesting that co-crystallization of ezetemibe with maleic acid has favored the dissolution of ezetemibe by molecular hydrogen bonding. It is speculated that maleic acids strong hydrogen bonding ability to ezetimibe might have increased the ability of co-crystal to interact with water molecules. We have concluded that dissolution was influenced by the co-former and also by changes in the particle size increasing surface area which contribute to faster dissolution. ${ }^{17}$

In anti-solvent method, both methanol and water are polar, both molecules exhibit hydrogen bonding where the hydrogen in one molecule is weakly covalently bonded to the oxygen in an adjacent molecule. ${ }^{14}$ The co-crystals prepared by this anti-solvent method demonstrated high solubility than that of pure drug because of the addition of anti solvent reduces the solute solubility in the resultant system. ${ }^{11}$ Out of the three co-formers used in this process, co-crystal prepared using maleic acid as a co-former showed better solubility than that of oxalic and succinic acid. Maleic acid has a higher dipole moment which enables intermolecular bonding with water molecules. ${ }^{14}$ But the dissolution profile of maleic acid was decreased in this method.

Further the optimized formulation was evaluated for SEM, XRD and DSC.

The FTIR studies were done and the peak shifts strongly indicates the formation of hydrogen bond and other weak interactions between the ezetimibe and maleic acid.

SEM results indicated significant changes in the surface morphology due to formulation process. The drug-maleic acid co-crystals formed from conventional sized ezetimibe were irregular shaped flake structures, as shown in Figure 5 some of the crystals featured sharp needle edges while others had less defined edges and more irregular shape. Different surface morphology for co-crystals can be attributed to the particle size of API chosen for the formulations which in turn have influence on the crystal lattice during crystallization. The particle size of prepared co-crystals was found to be $226.4 \pm 53 \mathrm{~nm}$ and had a polydispersity index value of 0.540 indicating that the optimized formulation is polydiperse in nature. DSC analysis, it was observed that the thermograms of the co-crystals had shown melting point $\left(130^{\circ} \mathrm{C}\right)$ that is less than the API. This difference in pattern can be attributed to formation of eutectic, melting at a lower temperature than either of the two pure components $\operatorname{drug}\left(163.5^{\circ} \mathrm{C}\right)$ and according to the previous literature maleic acid is showing a melting temperature of $\left(142^{\circ} \mathrm{C}\right)$. The thermal behavior of co-crystals was distinct with a different melting temperature from that of pure compound this suggests the formation of new phase. So the co-crystals formed from the API may be promoting additional co-crystallization and therefore maximizing the yield of co-crystals.

XRD studies have shown an intense peak at $27.7^{\circ}$ which indicated the formation of a new co-crystal phase. The probable conclusion which can be drawn from the XRD results is that the process facilitates co-crystallization.

\section{CONCLUSION}

In the present study, Nano co-crystals of ezetimibe were prepared and evaluated using different methods (Solvent evaporation method and Anti solvent method) employing different ratios of co-formers. The formulations were optimized in comparison with solubility of pure drug. The combination of a suitable conformer (Maleic acid) and a simple methodology (Solvent evaporation process) has allowed us to obtain nano sized cocrystals with significantly increased solubility and dissolution, which contribute to enhanced oral bioavailability of poorly water soluble drugs.

\section{Future scope}

The formulation can be further developed as conventional tablets with enhanced dissolution and bioavailability studies need to be performed on animals.

\section{ACKNOWLEDGEMENT}

The authors would like to express their sincere gratitude and heartfelt thanks to G. Pulla Reddy College of Pharmacy, for providing the best possible facilities to carry out the work successfully.

\section{CONFLICT OF INTEREST}

The authors have no conflict of interest.

\section{REFERENCES}

1. Srujan KM, Anna B, Kanakaiah KM. Liquisolid Compacts: Novel Approach for the Enhancement of Solubility of Poorly Soluble Drugs. Indo Am J Pharm Res. 2013;3(6):4386-95.

2. Finholt $P$, Solvang $S$. Dissolution kinetics of drugs in human gastric juice- the role of surface tension. J Pharm Sci. 1968;57(8):1322-6.

3. Lin SL, Ming J, Lachman L. Interdependence of Physiological Surfactant and Drug Particle Size on the Dissolution Behavior of Water Insoluble Drugs. J Pharm Sci.1968;57(12):2143-6.

4. Yuan G, Hui Z, Jianjun Z. Enhanced Dissolution and Stability of Adefovir Dipivoxil by Co-crystal Formation. J Pharm Pharmacol. 2010;63(4):483-90.

5. Srinivas B, Bostrom D, Sitaram PV. Indomethacin-Saccharin Co-crystal Design, Synthesis and Preliminary Pharmaceutical Characterization. Pharm Res. 2008;25(3):530-41. DOI: 10.1007/S11095-007-9394-1.

6. Sander JRG, Bucar DK, Henry RF, Zhang GGZ, MacGillivray LR. Pharmaceutical Nano-Cocrystals: Sonochemical Synthesis by Solvent Selection and use of a Surfactant. Angewandte Chemie International Edition. 2010;49(40):7284-8.

7. Spitzer D, Risse B, Schnell F, Pichot V, Klaumunze M, Schaefe MR. Continuous Engineering of Nano Co-crystals for Medical and Energetic applications. Sci Rep. 2014;4:1-6.

8. Sheetal S, Shete AS, Doijad RC, Kadam SS, Patil VA, Yadav AV. Formulation and Solid State Characterization of Nicotinamide-Based Co-crystals of Fenofribate. Indian J Pharm Sci. 2015;77(3):328-34.

9. Chandramouli Y, Gandhimathi R, Rubia YB, Amaravathi V. Review on Co-crystal as an Approach With Newer Implication in Pharmaceutical Field. Int J Med Chem Analysis. 2012;2(2):91-100. 
10. Kumari S, Santanu K, Samrat M, Jinu I, Animesh G. Solubility Enhancement of Ezetimibe by a Co-crystal Engineering Technique. Cryst Growth Des. 2014;14(9):4475-86

11. Yadav AV, Shete AS, Dabke AP, Kulkarni PV, Sakhare SS.Co-crystals: A Novel Approach to Modify Physicochemical Properties of Active Pharmaceutical Ingredients. Indian J Pharm Sci. 2009;71(4):359-70.

12. Marco Giulietti and Andre Bernardo. Crystallization by Antisolvent Addition and Cooling. ISBN 978-953-51-07-576 2012:379-396.

13. Shuo Z. Physical Properties and Crystallization of Theophylline Co-crystals, KTH School of Chemical Science and Engineering. Licentiate Thesis in Chemical
Engineering. 2010. ISSN 1654

14. Tanvee Patole and Ashwini Deshpande. Co-Crystallization- A Technique for Solubility Enhancement. Int J Pharm Sci Res. 2014;5(9):3566-76.

15. Bhupinder SS. Pharmaceutical Co-crystals an Update. Int Bull Drug Res. 2012;1(2):24-39

16. Yogesh KN, Vilas AA, Santosh GS, Sunil BB. Solubility Enhancement of Nevirapine by Co-crystallisation Technique. J Pharm Res . 2015;9(8):556-61.

17. David PE, René H, DeDiego LH. Use of pharmaceutical salts and cocrystals to address the issue of poor solubility. Int J Pharm. 2012;453(1):88-100.

Article History: Submission Date : 10-03-2020; Revised Date : 04-05-2020; Acceptance Date : 16-05-2020.

Cite this article: Bhandari J, Kanswami N, Lakshmi PK. Nano Co-crystal Engineering Technique to Enhance the Solubility of Ezetimibe. J Young Pharm. 2020;12(2)Suppl:s10-s5. 